\title{
Roundtable
}

\section{Response to Wilkinson \& Tepfer's “Fitness and beyond: preparing for the arrival of GM crops with ecologically important novel characters". Fuzzy reasoning and unacceptable change: defining and assessing an ambiguous endpoint}

\author{
TOM HARWOOD* \\ Centre for Environmental Policy, Imperial College London, Silwood Park, Ascot, Berkshire SL5 7PY, UK
}

Keywords: fuzzy reasoning / environmental risk assessment / genetically modified crop / fitness

In the context of ecological risk assessment, we require an effective definition of the undesirable endpoint. These may be defined according to scientific, economic and amenity value sets. Unfortunately, since the processes we are dealing with are continuous over space and time and also dynamic (in that ecological population sizes fluctuate), unambiguous definitions are elusive. In the case of enhanced fitness, an organism that is fitter than a direct competitor will continuously increase its numbers at the expense of its competitor. Unless a change in fitness or environment occurs, the competitor will often eventually be driven to local extinction. It could therefore be argued that any enhanced fitness is undesirable.

We can be concerned about the decline of a particular species, assemblage or visual landscape, but at what point does it become unacceptable? At one extreme, one could argue that any decline is unacceptable. At the other, a definite endpoint would be the "rapid" extinction of whatever we are trying to protect. In between, we have a shift in the ecological balance, leading to a continuous decline of the protectee. Here we have to define a timescale, and some form of measurable degree of change. Whilst we can ignore the problem and use true but unhelpful statements such as "every situation will have its own unique criteria", this will not lead to a satisfactory definition. Instead, we require a concrete framework to deal with what is essentially a qualitative judgement.

It seems to me that the crux of the problem lies in its inherent ambiguity. Acceptability is continuous in itself. An ecological situation will become less acceptable and more unacceptable as it moves in the undesirable

*Corresponding author: t.harwood@imperial.ac.uk direction, but at no clear point does a testable endpoint occur. We could attempt to bypass this problem by defining each endpoint deep into the unacceptable category, e.g. $50 \%$ decline in abundance over 10 years, but what about $50 \%$ decline in 11 years, or $49 \%$ in 10 years?

I believe that the solution to this problem lies with fuzzy logic (Zadeh, 1965), or approximate reasoning, (even though fuzzy set theory wasn't very constructive when applied to populations (Harwood, 2009)). This allows one to reason with ambiguous concepts, shades of grey rather than black and white. For example, one could define a functional relationship to describe the decline in acceptability with abundance and time. Fuzzy reasoning is well established (see Cox, 1999, for practical implementation) and used widely in commercial applications (both in control systems and economic risk assessment), but often misrepresented by ignorant (or partially ignorant) theoreticians who view it as a probabilistic issue (i.e. it is simply Bayesian logic in disguise). We can refute this with a simple example. In fuzzy set theory, an apple can have different degrees of redness and greenness. In Boolean logic, it can only be red or green. Applying probability to this, an apple which is half red and half green could be said to have a probability of 0.5 of being green. This is clearly nonsense since it is what it is. Similarly, the $49 \%$ decline in 11 years does not have a probability of being acceptable, rather it has a degree of acceptability: we can say it is barely acceptable or nearly unacceptable.

It is straightforward to combine Bayesian reasoning, based on uncertainty of inputs/outcomes, etc., with fuzzy reasoning, using ambiguous concepts such as risk. I therefore believe that this presents a feasible solution 
to the dilemma which we face in biosafety regulation. In each case, one can build a definition of unacceptable change using expert opinion (scientific and sociopolitical). This will allow us to quantify risk in terms of the fuzzy endpoint. If required, a fuzzy system can deliver Boolean outputs: Yes it will be OK: No it will be bad: but is probably more useful if it delivers a qualitative assessment of the degree of risk, e.g. minimal risk, low risk, moderate risk, high risk, very high risk.

My feeling is that unless we address this fundamental issue (possibly by another means), we will always end up going round in circles.
Received January 28, 2009; accepted March 11, 2009.

\section{REFERENCES}

Cox E (1999) The Fuzzy Systems Handbook: a practitioner's guide to building, using and maintaining fuzzy systems, Academic Press Professional, Chappaqua, New York

Harwood TD (2009) The circular definition of populations and its implication for biological sampling. Mol. Ecol. 18: 765-768

Zadeh LA (1965) Fuzzy sets. Inform. Control 8: 338-353 